\title{
PAPEL DE LAS LOMBRICES DE TIERRA EN LA DEGRADACIÓN DEL BAGAZO DE UVA: EFECTOS SOBRE LAS CARACTERÍSTICAS QUÍMICAS Y LA MICROFLORA EN LAS PRIMERAS ETAPAS DEL PROCESO
}

\author{
María Gómez-Brandón, ${ }^{1}$ Cristina LazCano, ${ }^{1}$ Marta LoRes $^{2}$ \& Jorge \\ DOMÍNGUEz ${ }^{1}$ \\ ${ }^{1}$ Departamento de Ecología y Biología Animal. Universidad de Vigo. Vigo E-36310, España. E-mail: \\ mariagomez@uvigo.es, cristina@uvigo.es, jdguez@uvigo.es \\ ${ }^{2}$ Departamento de Química Analítica, Nutrición y Bromatología, Facultad de Química. Avda. de las \\ Ciencias s/n, Campus Sur E-15782, Santiago de Compostela, España. E-mail: qnmlores@usc.es,
}

Gómez-Brandón, M., C. Lazcano, M. Lores \& J. Domínguez. 2010. Papel de las lombrices de tierra en la degradación del bagazo de uva: efectos sobre las características químicas y la microflora en las primeras etapas del proceso. Acta Zoológica Mexicana (n.s.), Número Especial 2: 397-408.

RESUMEN. La industria vitivinícola, de gran importancia en varias regiones de España, genera una gran cantidad de residuos originados en las distintas etapas de la fabricación del vino. De las diversas alternativas para su tratamiento, el vermicompostaje constituye un proceso adecuado de estabilización de estos residuos, eliminando su contaminación potencial y permitiendo obtener un producto estable e inocuo con grandes posibilidades de utilización en la agricultura. En este trabajo estudiamos los cambios químicos, bioquímicos y microbiológicos que se producen a corto plazo en la degradación del bagazo de uva, un residuo orgánico cuya acumulación y gestión constituye un importante problema ambiental. Tras quince días de procesado por las lombrices, se observó una reducción significativa en la biomasa fúngica medida como el contenido de ergosterol, así como una disminución de la actividad microbiana y de las actividades enzimáticas celulasa y proteasa. La rapidez con que ocurrieron estas transformaciones hacen del proceso de vermicompostaje un buen sistema para estudiar el papel de las lombrices de tierra y su microflora asociada en la primeras etapas de la degradación del bagazo de uva; y proporcionan un importante avance de la posible aplicación del vermicompostaje como una alternativa para el tratamiento de residuos orgánicos derivados de la industria vitivinícola.

Palabras clave: Vermicompostaje, bagazo de uva, respiración basal, ergosterol, actividades enzimáticas.

Gómez-Brandón, M., C. Lazcano, M. Lores \& J. Domínguez. 2010. Role of earthworms in the degradation of grape marc: effects on chemical characteristics and microflora in the first steps of the process. Acta Zoológica Mexicana (n.s.), Número Especial 2: 397-408.

ABSTRACT. The wine industry, of particular importance in various regions of Spain, generates vast amounts of organic waste during the different stages of wine production. Among the possible methodological alternatives available for its treatment, vermicomposting is one of the best-known processes for 
the biological stabilization of solid organic wastes by transforming them into safer and more stabilized materials suitable for application to soil. In this study we analyzed the chemical, biochemical and microbiological changes that occur in the first stages of the degradation of grape marc, an organic residue whose accumulation and management constitute an important environmental problem. After fifteen days of vermicomposting, a significant reduction in the fungal biomass measured as the ergosterol content was observed, as well as a reduction in microbial activity and cellulase and protease activities. The speed at which these transformations occurred makes vermicomposting a good system for studying the role of earthworms and their associated microflora in the first stages of grape marc degradation, providing important advances in the possible application of vermicomposting as an alternative for the treatment of organic residues derived from the wine industry.

Keywords: Vermicomposting, grape marc, basal respiration, ergosterol, enzymatic activities.

\section{INTRODUCCIÓN}

El bagazo es un residuo orgánico derivado de la industria vitivinícola formado por los restos sólidos que quedan después de la extracción del mosto de los racimos de uvas. En España se producen más de 750.000 toneladas al año de bagazo (Fernández-Bayo et al. 2007) y su acumulación y gestión constituye un importante problema ambiental. Una solución para la reutilización de este subproducto rico en polisacáridos es su empleo como enmienda orgánica, ya que es rico en nutrientes, principalmente nitrógeno y potasio, esenciales para el crecimiento de las plantas y el desarrollo de los cultivos (Bertran et al. 2004). Sin embargo, su utilización directa e indiscriminada es problemática, ya que puede liberar un exceso de taninos y fenoles en el suelo que pueden inhibir el crecimiento de las raíces (Inbar et al. 1991).

El compostaje y el vermicompostaje son dos alternativas metodológicas muy eficientes para el tratamiento de residuos orgánicos sólidos y pueden eliminar el riesgo contaminante de los residuos y convertirlos en biofertilizantes y bioplaguicidas con grandes posibilidades de utilización en la agricultura (Domínguez et al., este número). Mientras que el compostaje ha sido empleado con éxito en el tratamiento de residuos de la industria vitivinícola (Inbar et al. 1992, Ferrer et al. 2001, Diaz et al. 2002, Bertran et al. 2004, Flavel et al. 2005), existen pocos estudios de la posible aplicación del vermicompostaje para el reciclaje de los mismos (Nogales et al. 2005, Romero et al. 2007).

El vermicompostaje es un proceso de bio-oxidación, degradación y estabilización de la materia orgánica desarrollado por la acción conjunta y sinérgica de las lombrices de tierra y los microorganismos. Durante este proceso los sustratos orgánicos se transforman a través de dos fases, una inicial, denominada fase activa, en la que las lombrices fragmentan y acondicionan el sustrato incrementando el área expuesta a la actividad microbiana, y alterando su actividad biológica de forma importante; las lombrices son, por tanto, agentes cruciales del proceso al actuar como facilitadores clave de las transformaciones de la materia orgánica con efectos directos sobre la tasa de descomposición y la calidad de los productos finales. La segunda fase es una etapa de 
maduración, caracterizada por el desplazamiento de las lombrices hacia capas nuevas con residuo fresco y por la actuación de poblaciones microbianas más especializadas, responsables de la degradación de polímeros complejos como la lignina. La duración de esta etapa depende de la complejidad del material de partida y de la eficacia con la que se haya desarrollado la fase activa del proceso (Domínguez 2004).

El vermicompost, que es el producto final del vermicompostaje, tiene valor como enmienda orgánica del suelo: es un material estabilizado, homogéneo, rico en nutrientes y de granulometría fina, con una baja relación $\mathrm{C} / \mathrm{N}$, una porosidad alta $\mathrm{y}$ una elevada capacidad de retención de agua. La adición de vermicompost al suelo aumenta su porosidad y la retención de humedad e incrementa la disponibilidad de nutrientes para las plantas (Domínguez et al., este número). Además, equilibra la microflora y la microfauna del suelo, inhibiendo o reduciendo las ventajas de determinados patógenos oportunistas (Domínguez et al., este número).

El conocimiento del proceso de vermicompostaje y de los mecanismos biológicos que lo rigen es fundamental para su desarrollo no sólo como alternativa metodológica para el tratamiento de residuos orgánicos sino también para obtener un producto con características fertilizantes y plaguicidas, medioambientalmente adecuado y de alta calidad como enmienda orgánica. El objetivo de este trabajo fue conocer qué tipos de cambios químicos, bioquímicos y microbiológicos se producen durante la fase activa del proceso de vermicompostaje del bagazo de uva, y así ampliar nuestro conocimiento del papel que desempeñan las lombrices de tierra en el proceso de descomposición de la materia orgánica.

\section{MATERIAL Y MÉTODOS}

El bagazo de uva se recogió en una destilería de la provincia de Pontevedra (Galicia, España) y se almacenó a $5{ }^{\circ} \mathrm{C}$ en una cámara fría hasta el momento de su utilización. Se trata de un sustrato rico en polisacáridos y por lo tanto, se esperaba una respuesta rápida de las lombrices de tierra y de los microorganismos al tener a su disposición una gran cantidad de compuestos carbonados de fácil asimilación.

\section{Diseño experimental}

El bagazo, previamente aireado y humedecido, se sometió a un proceso de vermicompostaje con la especie de lombriz de tierra Eisenia andrei. Se emplearon recipientes plásticos de $24 \mathrm{~cm}$ de longitud, $12 \mathrm{~cm}$ de altura y $11 \mathrm{~cm}$ de lado que se llenaron hasta $3 / 4$ partes de su capacidad con una cama de vermicompost maduro para asegurar la supervivencia de las lombrices $(n=5)$. Se introdujeron individuos juveniles y adultos ( $250 \mathrm{~g}$ por recipiente); sobre la superficie del sustrato se dispuso una red de plástico con $5 \mathrm{~mm}$ de luz de malla sobre la que se depositó el bagazo (1 $\mathrm{kg}$ por recipiente). Los recipientes se mantuvieron en una cámara de cultivo a $25 \pm 2{ }^{\circ} \mathrm{C}$ durante 15 días. 
Transcurrido este tiempo se retiraron las lombrices de los mesocosmos, y se procedió a la toma de muestras; la biomasa de lombrices al cabo de los 15 días no varió con respecto a la inicial. El tiempo de incubación depende de la especie de lombriz elegida para el vermicompostaje, y de la densidad de lombrices presentes en el residuo. $E$. andrei es una especie epigea con una tasa alta de consumo, digestión y asimilación de la materia orgánica; por tanto, fueron suficientes quince días para que esta especie procesase el bagazo completamente.

Se incluyó también un control que consistió en la disposición del bagazo en recipientes plásticos de igual dimensión a los empleados para el vermicompostaje $(n=5)$, y en la incubación de estos recipientes en la cámara de cultivo $\left(25 \pm 2^{\circ} \mathrm{C}\right)$ durante 15 días. Al cabo de este tiempo se procedió a la toma de muestras.

Todos los sustratos (bagazo inicial, control y vermicompost) se tamizaron (5 mm) para la eliminación de las semillas de las uvas y los rabos de los racimos.

\section{Análisis químicos, bioquímicos y microbiológicos de las muestras}

La humedad se determinó mediante el método gravimétrico por pérdida de peso a 105 ${ }^{\circ} \mathrm{C}$ durante 24 horas; y el contenido de materia orgánica por ignición tras la calcinación de la muestra seca a $550 \pm 50{ }^{\circ} \mathrm{C}$ durante 4 horas en un horno-mufla. $\mathrm{El} \mathrm{pH}$ y la conductividad eléctrica se midieron en extractos de agua destilada en proporción 1:10 (peso fresco:volumen). La concentración de $\mathrm{C}$ y $\mathrm{N}$ total se determinó en muestra seca con un analizador elemental Carlo Erba $1500 \mathrm{C} / \mathrm{N}$. El N inorgánico $\left(\mathrm{NH}_{4}^{+} \mathrm{y} \mathrm{NO}_{3}{ }^{-}\right)$se midió en extractos de $\mathrm{KCl} 2 \mathrm{~N}$ por valoración ácido-base con $\mathrm{HCl} 0,01 \mathrm{~N}$ utilizando un destilador Büchi. El análisis de fibras (celulosa, hemicelulosa y lignina) se realizó según el método fibra detergente propuesto por Goering \& Van Soest (1970).

$\mathrm{El} \mathrm{C}$ de la biomasa microbiana se midió en extractos de $\mathrm{K}_{2} \mathrm{SO}_{4} 0,5 \mathrm{M}$ en proporción 1:50 (peso fresco:volumen) según el método de fumigación-extracción (Vance et al. 1987). La actividad microbiana se cuantificó midiendo la tasa de producción de $\mathrm{CO}_{2}$ después de 6 de incubación a temperatura ambiente con trampas de $\mathrm{NaOH}$ (Aira et al. 2007a).

El ergosterol es uno de los principales esteroles presentes en la membrana de los hongos Ascomicetos, Basidiomicetos y hongos imperfectos y se utiliza como biomarcador fúngico (Frostegård \& Bååth 1996). La extracción del ergosterol se realizó con microondas (Young 1995) y su concentración se determinó mediante HPLC (Aira et al. 2007a).

El sistema enzimático celulasa cataliza la hidrólisis de la celulosa a D-glucosa mediante una secuencia de reacciones, y consiste en al menos tres enzimas: endo- $\beta-$ 1,4-glucanasas, exo- $\beta$-1,4-glucanasas y $\beta$-glucosidasas. El método utilizado para el análisis de este sistema enzimático se basa en la determinación de los azúcares reducidos, liberados después de incubar la muestra 24 horas a $50{ }^{\circ} \mathrm{C}$ con una solución de 
la sal sódica de la carboximetil celulosa (Schinner \& Von Mersi 1990).

El enzima proteasa cataliza la hidrólisis de compuestos nitrogenados (proteínas y péptidos) a nitrógeno amoniacal. El método utilizado para el análisis de este enzima se basa en la determinación de los aminoácidos liberados después de incubar la muestra 2 horas a $50^{\circ} \mathrm{C}$ con caseinato sódico, empleando el reactivo Folin- Ciocalteu (Ladd \& Butler 1972).

\section{Análisis estadístico}

Se aplicó un análisis de varianza univariante (ANOVA), y un test a posteriori HSD de Tukey (Tukey Honestly Significant Difference) utilizando el paquete estadístico SPSS versión 11.5. Todas las variables cumplieron los criterios de normalidad y homocedasticidad necesarios para la realización de estos análisis.

\section{RESULTADOS}

En nuestro estudio, el bagazo de uva inicial presentó $70 \%$ de humedad; tras 15 días de incubación no se observó un cambio significativo en este parámetro (Cuadro I). El contenido de materia de orgánica del bagazo de uva fue de $93 \%$, y su valor se redujo ligeramente (pero no de forma significativa, tras 15 días de incubación (Cuadro I).

Cuadro I. Propiedades químicas (media \pm error estándar, $n=5$ ) del bagazo de uva inicial, y de los sustratos obtenidos tras 15 días de incubación en ausencia de lombrices (control) y en presencia de

Eisenia andrei (vermicompost). Las diferencias significativas entre los valores obtenidos para los distintos sustratos (inicial, control y vermicompost) se representan con diferentes letras $(p<0,05$, test HSD de Tukey).

\begin{tabular}{lccccc}
\hline \multicolumn{1}{c}{ Parámetros químicos } & Bagazo inicial & Control & Vermicompost & \multicolumn{2}{c}{ ANOVA } \\
\cline { 3 - 6 } & & & & $\mathrm{F}$ & $\mathrm{P}$ \\
\hline Humedad (\%) & & & $75 \pm 1 \mathrm{a}$ & 0.440 & 0.654 \\
Materia orgánica (\%) & $93 \pm 1 \mathrm{a}$ & $90 \pm 1 \mathrm{a}$ & $85 \pm 1 \mathrm{a}$ & 2.206 & 0.153 \\
Conductividad eléctrica $\left(\mathrm{mS} \mathrm{cm}^{-1}\right)$ & $0.28 \pm 0.01 \mathrm{a}$ & $0.26 \pm 0.01 \mathrm{a}$ & $0.27 \pm 0.01 \mathrm{a}$ & 0.339 & 0.719 \\
$\mathrm{pH}$ & $7.77 \pm 0.01 \mathrm{~b}$ & $7.77 \pm 0.03 \mathrm{~b}$ & $8.12 \pm 0.09 \mathrm{a}$ & 14.083 & 0.001 \\
Total C $\left(\mathrm{g} \mathrm{kg}^{-1}\right)$ & $503 \pm 2 \mathrm{a}$ & $486 \pm 3 \mathrm{a}$ & $459 \pm 6 \mathrm{~b}$ & 13.624 & 0.001 \\
Total N $\left(\mathrm{g} \mathrm{kg}^{-1}\right)$ & $35 \pm 3 \mathrm{~b}$ & $47 \pm 1 \mathrm{a}$ & $47 \pm 2 \mathrm{a}$ & 6.084 & 0.015 \\
Relación C/N & $14.4 \pm 1.44 \mathrm{a}$ & $10.3 \pm 0.24 \mathrm{~b}$ & $9.8 \pm 0.46 \mathrm{~b}$ & 5.831 & 0.017 \\
$\mathrm{NH}_{4}^{+}\left(\mathrm{mg} \mathrm{kg}^{-1}\right.$ peso seco) & $170 \pm 13 \mathrm{ab}$ & $127 \pm 12 \mathrm{~b}$ & $193 \pm 21 \mathrm{a}$ & 6.787 & 0.011 \\
$\mathrm{NO}_{3}^{-}\left(\mathrm{mg} \mathrm{kg}^{-1}\right.$ peso seco) & $78 \pm 6 \mathrm{a}$ & $80 \pm 6 \mathrm{a}$ & $80 \pm 4 \mathrm{a}$ & 1.329 & 0.301 \\
Celulosa $\left(\mathrm{g} \mathrm{kg}^{-1}\right)$ & $175 \pm 4 \mathrm{a}$ & $169 \pm 4 \mathrm{ab}$ & $148 \pm 5 \mathrm{~b}$ & 8.847 & 0.004 \\
Hemicelulosa $\left(\mathrm{g} \mathrm{kg}^{-1}\right)$ & $69 \pm 5 \mathrm{a}$ & $51 \pm 8 \mathrm{ab}$ & $40 \pm 6 \mathrm{~b}$ & 7.447 & 0.008 \\
Lignina $\left(\mathrm{g} \mathrm{kg}^{-1}\right)$ & $517 \pm 3 \mathrm{a}$ & $531 \pm 14 \mathrm{a}$ & $543 \pm 8 \mathrm{a}$ & 3.985 & 0.067 \\
\hline
\end{tabular}


La conductividad eléctrica del bagazo inicial fue $0,28 \mathrm{mS} \mathrm{cm}^{-1}$, y no se observó un cambio significativo en este parámetro tras 15 días de incubación (Cuadro I). El $\mathrm{pH}$ del bagazo inicial fue 7.77 y varió de forma significativa tras 15 días de incubación (Cuadro I). El vermicompostaje aumentó significativamente el $\mathrm{pH}$ del bagazo (hasta 8,12) (Cuadro I).

El contenido de $\mathrm{C}$ total del bagazo inicial fue $503 \mathrm{~g} \mathrm{~kg}^{-1}$, y varió de forma significativa tras 15 días de incubación, con menor valor $\left(459 \mathrm{~g} \mathrm{~kg}^{-1}\right)$ tras el vermicompostaje (Cuadro I). El bagazo inicial presentó un contenido de $\mathrm{N}$ total de $35 \mathrm{~g} \mathrm{~kg}^{-1} \mathrm{y}$ al igual que el $\mathrm{C}$ total, su concentración varió de forma significativa tras 15 días de incubación (Cuadro I). Sin embargo, contrario a lo esperado, el contenido de $\mathrm{N}$ total fue significativamente mayor en el sustrato control y el vermicompost $\left(47 \mathrm{~g} \mathrm{~kg}^{-1}\right)$. La relación $\mathrm{C} / \mathrm{N}$ del bagazo inicial fue 14.4, y tras 15 días de incubación se observó un cambio significativo en esta relación (Cuadro I), siendo por debajo de 11 tanto en el sustrato control como en el vermicompost.

El contenido de nitrógeno amoniacal del bagazo inicial fue $170 \mathrm{mg} \mathrm{kg}^{-1}$ peso seco, y varió de forma significativa tras 15 días de incubación (Cuadro I). La concentración de $\mathrm{NH}_{4}^{+}$del bagazo se redujo hasta $127 \mathrm{mg} \mathrm{kg}^{-1}$ peso seco en el sustrato control debido muy probablemente a las pérdidas de $\mathrm{N}$ por volatilización, aunque estas diferencias no fueron significativas (Cuadro I); tras el vermicompostaje se detectó, sin embargo, una concentración significativamente mayor $\left(193 \mathrm{mg} \mathrm{kg}^{-1}\right)($ Cuadro I). El contenido de nitratos del bagazo inicial fue $78 \mathrm{mg} \mathrm{kg}^{-1}$ peso seco, y no se observó un cambio significativo en este parámetro tras 15 días de incubación (Cuadro I).

El contenido de celulosa y hemicelulosa del bagazo inicial fue 175 y $69 \mathrm{~g} \mathrm{~kg}^{-1}$, respectivamente; su concentración varió de forma significativa tras 15 días de incubación (Cuadro I), registrándose los valores más bajos en el vermicompost. En cuanto al contenido de lignina, el bagazo inicial presentó un valor de $517 \mathrm{~g} \mathrm{~kg}^{-1}, \mathrm{y}$ tras 15 días de incubación no se registraron cambios significativos en su concentración (Cuadro I).

El carbono de la biomasa microbiana del bagazo inicial fue $38490 \mathrm{mg} \mathrm{kg}^{-1}$ peso seco; tras 15 días de incubación se observó un cambió significativo en su concentración (Fig. 1A, ANOVA $F_{2,12}=7.736 ; \mathrm{P}=0.047$ ), registrándose valores más elevados en el sustrato control y el vermicompost (57,264 y 49,338 $\mathrm{mg} \mathrm{kg}^{-1}$, respectivamente) aunque sólo se encontraron diferencias significativas entre el bagazo inicial y el sustrato control (Fig. 1A). La biomasa fúngica medida como el contenido de ergosterol fue $33 \mathrm{mg} \mathrm{kg}^{-1}$ materia orgánica en el bagazo inicial, y tras 15 días de incubación su concentración varió de forma significativa (Fig. $1 \mathrm{~B}$, ANOVA $_{2,12}=46,648 ; \mathrm{P}=$ 0.001 ), disminuyendo significativamente (hasta $6 \mathrm{mg} \mathrm{kg}^{-1}$ ) tras el vermicompostaje (Fig. 1B).

La actividad microbiana medida como respiración basal fue $6326 \mathrm{mg} \mathrm{CO}_{2} \mathrm{~kg}^{-1}$ materia orgánica en el bagazo inicial. Tras 15 días de incubación se observó un cambio significativo en este parámetro (Fig. 2, ANOVA $\mathrm{F}_{2,12}=110,672 ; \mathrm{P}=0.001$ ); la 


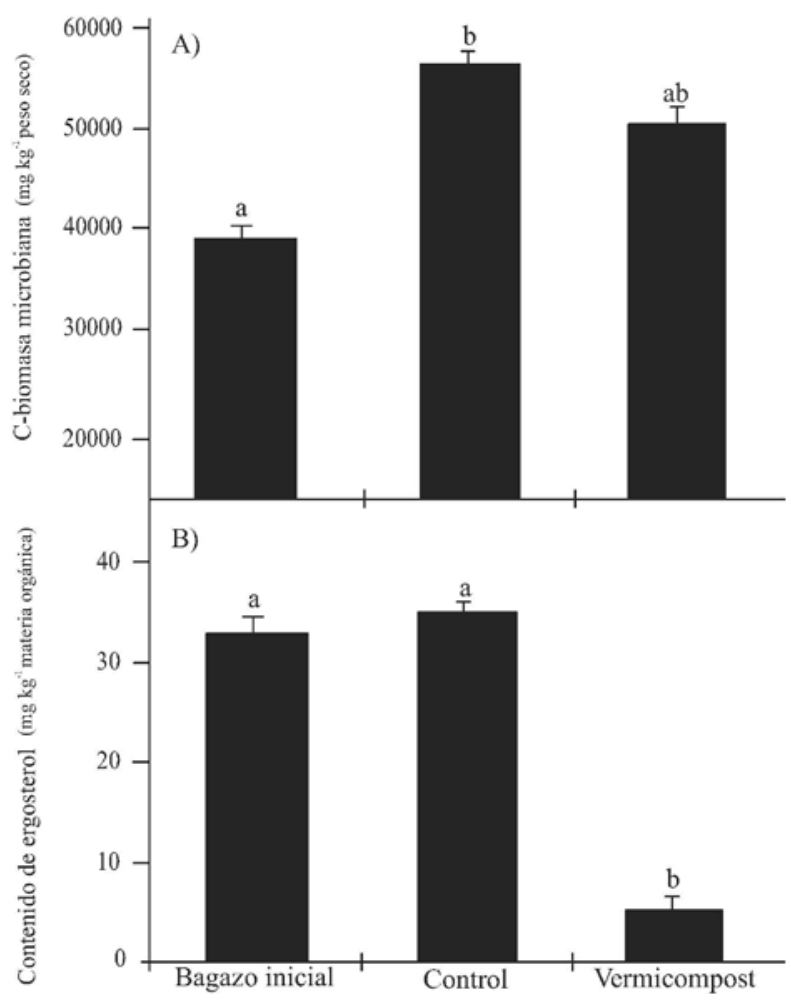

Figura 1. Valores (media \pm error estándar, $n=5$ ) del C-biomasa microbiana (A) y del contenido de ergosterol (B) del bagazo de uva inicial, y de los sustratos obtenidos tras 15 días de incubación en ausencia de lombrices (control) y en presencia de Eisenia andrei (vermicompost). Las diferencias significativas entre los valores obtenidos para los distintos sustratos (inicial, control y vermicompost) se representan con diferentes letras $(\mathrm{P}<0.05$, test HSD de Tukey).

actividad microbiana del bagazo se redujo considerablemente en el sustrato control (4842 $\mathrm{mg} \mathrm{CO}_{2} \mathrm{~kg}^{-1}$ ), aunque ésta fue mucho más baja tras el vermicompostaje (2795 $\left.\mathrm{mg} \mathrm{CO} \mathrm{kg}^{-1}\right)$.

La actividad celulasa del bagazo inicial fue $8220 \mathrm{mg}$ eq glucosa $\mathrm{g}^{-1}$ peso seco, y varió de forma significativa tras 15 días de incubación (Fig. 3, ANOVA $\mathrm{F}_{2,12}=$ 10,158; $\mathrm{P}=0,003)$. La actividad de este enzima aumentó ligeramente en el sustrato control y alcanzó un valor de $8550 \mathrm{mg}$ eq glucosa $\mathrm{g}^{-1}$ peso seco; tras el vermicompostaje se detectó, sin embargo, una actividad mucho menor $(5850 \mathrm{mg}$ eq glucosa $\mathrm{g}^{-1}$ ), de forma que el vermicompost fue significativamente diferente en cuanto a su actividad celulasa del bagazo inicial y del sustrato control (Fig. 3). El bagazo inicial presentó una actividad proteasa de $8108 \mathrm{mg}$ tirosina $\mathrm{kg}^{-1}$ peso seco. Tras 15 días de incubación se observaron los mismos fenómenos observados para a actividad de la 


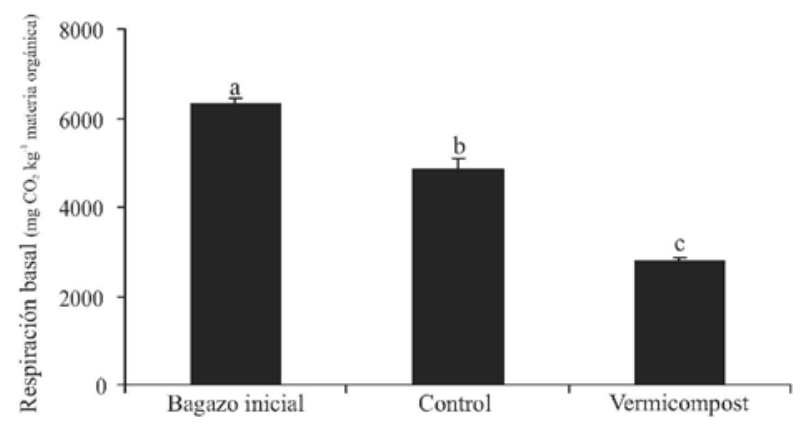

Figura 2. Valores (media \pm error estándar, $n=5$ ) de la actividad microbiana medida como respiración basal del bagazo de uva inicial, y de los sustratos obtenidos tras 15 días de incubación en ausencia de lombrices (control) y en presencia de Eisenia andrei (vermicompost). Las diferencias significativas entre los valores obtenidos para los distintos sustratos (inicial, control y vermicompost) se representan con diferentes letras $(\mathrm{P}<0.05$, test HSD de Tukey).

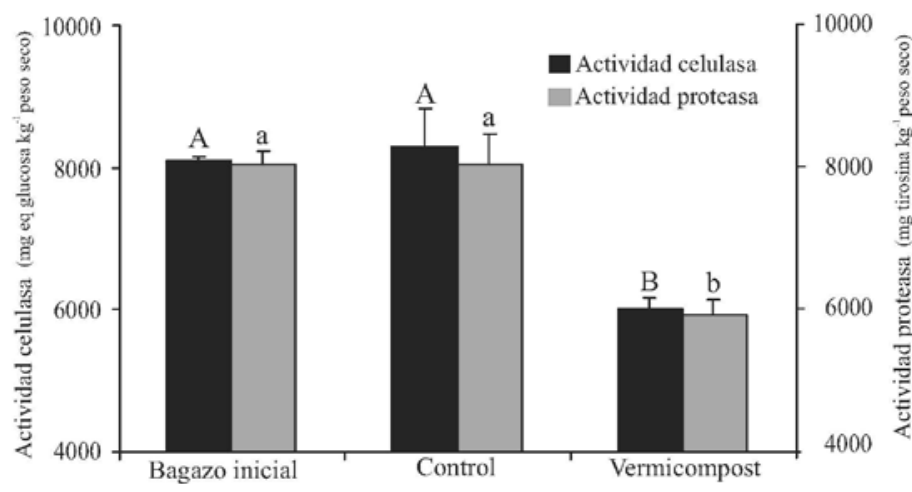

Figura 3. Valores (media \pm error estándar, $n=5$ ) de las actividades enzimáticas celulasa y proteasa del bagazo de uva inicial, y de los sustratos obtenidos tras 15 días de incubación en ausencia de lombrices (control) y en presencia de Eisenia andrei (vermicompost). Las diferencias significativas entre los valores obtenidos para los distintos sustratos (inicial, control y vermicompost) se representan con diferentes letras $(\mathrm{P}<0.05$, test HSD de Tukey).

celulasa, con valores significativamente menores tras el vermicompostaje y sin diferencias significativas entre el bagazo inicial y el sustrato control (Fig. 3).

\section{DISCUSIÓN}

El contenido de humedad del sustrato es uno de los factores limitantes para el desarrollo de las lombrices de tierra durante el proceso de vermicompostaje (Edwards \& Bohlen 1996). Los óptimos de humedad para el crecimiento y desarrollo de la lombriz E. andrei están entre el 80 y el 90\% (Domínguez \& Edwards 1997), valores 
ligeramente mayores que los encontrados en los sustratos del presente estudio. La conductividad eléctrica es una medida de la concentración de sales en el medio y al igual que la humedad se considera un factor limitante para la supervivencia de las lombrices de tierra. Así, concentraciones elevadas de sales en los residuos pueden tener efectos inhibitorios en el desarrollo y la reproducción de las lombrices, e incluso pueden causar su muerte. Con respecto a $E$. andrei, valores de conductividad superiores a $8 \mathrm{dS} \mathrm{m}^{-1}$ se consideran letales (Edwards 1988). En nuestro estudio los valores de conductividad obtenidos en el vermicompost no superaron este límite. El mayor valor de $\mathrm{pH}$ encontrado en el vermicompost podría explicarse por la aparición, como consecuencia de la acción de las lombrices, de una microflora metabólicamente más activa, en el residuo que, a lo largo de los 15 días, degradó compuestos orgánicos lábiles como aminoácidos, resultando en la liberación de nitrógeno amoniacal, y por tanto en la alcalinización del residuo.

Durante el proceso de vermicompostaje, una fracción de la materia orgánica contenida en los residuos se mineraliza, por lo que los valores de carbono orgánico total, y por tanto la relación $\mathrm{C} / \mathrm{N}$ se reducen de forma notable. También se observó una menor concentración de celulosa y hemicelulosa, pero en este caso las diferencias con respecto al control no fueron significativas. Estos resultados afianzan la hipótesis de que las lombrices de tierra aceleran la tasa de descomposición de la materia orgánica durante el proceso de vermicompostaje, tal y como han señalado otros autores (Domínguez et al. 2010). A diferencia del pool de carbono, se detectó un incremento en el contenido de nitrógeno total en ambos tratamientos con y sin lombrices. Este incremento podría deberse a un efecto de concentración del mismo como consecuencia de la mineralización de la materia orgánica, que produce la pérdida de otros elementos como el carbono en forma de $\mathrm{CO}_{2}$. La actividad de las lombrices epigeas también aumentó la concentración de $\mathrm{NH}_{4}{ }^{+}$en comparación con el control debido muy probablemente a que el $\mathrm{NH}_{4}{ }^{+}$es uno de sus productos de excreción (Lee 1985). Se ha demostrado que las lombrices favorecen la nitrificación, que resulta en la conversión rápida del nitrógeno amoniacal en nitratos, aumentando la mineralización de nitrógeno. En este sentido, Atiyeh et al. (2000) y Domínguez (2004) registraron un aumento en la concentración de $\mathrm{NO}_{3}{ }^{-}$en un experimento de vermicompostaje con estiércol de vaca y purín de cerdo, respectivamente. Sin embargo, en nuestro estudio quince días de vermicompostaje no fueron suficientes para detectar un incremento en el contenido de nitratos del bagazo, y por tanto, con respecto a este parámetro, sería necesario haber estudiado las fases posteriores del proceso.

Dichos cambios en la composición química del residuo afectaron en gran medida a la biomasa microbiana y su actividad. Así, tras quince días de procesado por las lombrices epigeas, la biomasa fúngica disminuyó con respecto al control. Esto podría ser debido al consumo de los hongos por las lombrices (Doube \& Brown 1998) o a una menor disponibilidad de compuestos carbonados tales como la celulosa tras el 
proceso de vermicompostaje. El incremento en la biomasa microbiana total al cabo de los 15 días podría deberse a que la estimación de $\mathrm{C}$ por el método de fumigaciónextracción se considera una medida absoluta, es decir, no se trata de un método de extracción selectivo a diferencia del análisis de los ácidos grasos unidos a fosfolípidos que proporciona información sobre la biomasa viva y activa, o la respiración inducida por sustrato que sólo hace referencia a la biomasa sensible a sustratos lábiles como la glucosa.

Tras quince días de procesado por las lombrices epigeas la actividad microbiana total del bagazo de uva se redujo en mayor medida que en el tratamiento control, tal y como han señalado y encontrado otros autores previamente (Aira et al. 2002, 2006, Aira \& Domínguez 2009). También se detectó un descenso en la actividad de las dos enzimas analizadas, mientras que en el tratamiento control no se observó tal reducción. Aira et al. (2006) también observaron una disminución de la actividad proteasa de purín de cerdo en presencia de la lombriz epigea Eudrilus eugeniae, mientras que no detectaron un cambio significativo en la actividad celulasa. Las actividades enzimáticas han sido propuestas como índices de calidad del sustrato por el control que ejercen en la dinámica de la materia orgánica y en la liberación de nutrientes para el crecimiento vegetal y microbiano (Dick et al. 1996). En los últimos años, se han utilizado diferentes actividades enzimáticas como biomarcadores del proceso de vermicompostaje (Benítez et al. 2002, Benítez et al. 2005, Aira et al. 2007b) para obtener información acerca de los cambios de los residuos orgánicos, así como del funcionamiento del ciclo de nutrientes. A medida que avanza el proceso de vermicompostaje $\mathrm{y}$, consecuentemente, la biodegradación de la materia orgánica por la acción conjunta de las lombrices de tierra y los microorganismos, la mayor parte de las actividades enzimáticas estudiadas tienden a disminuir debido a una menor disponibilidad de sustrato.

La actividad de la lombriz epigea $E$. andrei aceleró la descomposición del bagazo de uva después de dos semanas de vermicompostaje. El menor contenido de $\mathrm{C}$ total encontrado en el vermicompost, respecto al bagazo inicial y al sustrato control y la reducción significativa en la concentración de celulosa y hemicelulosa tras el vermicompostaje evidencian el papel de las lombrices de tierra como facilitadores clave de las transformaciones de la materia orgánica con efectos directos sobre la tasa de descomposición del sustrato. La biomasa fúngica, la actividad microbiana, medida como respiración basal, y las actividades enzimáticas celulasa y proteasa también disminuyeron tras quince días de vermicompostaje con respecto al bagazo inicial y al sustrato control. La rapidez con que ocurrieron estas transformaciones químicas, bioquímicas y microbianas hacen del proceso de vermicompostaje un buen sistema para estudiar el papel que desempeñan las lombrices de tierra en la descomposición la materia orgánica a través de sus relaciones con los microorganismos, y proporcionan un importante avance de la posible aplicación del vermicompostaje como alternati- 
va metodológica para el tratamiento de residuos orgánicos derivados de la industria vitivinícola.

\section{AGRADECIMIENTOS}

Este trabajo ha sido financiado por la Xunta de Galicia (proyectos 07MRU023383PR y 09TAL012209PR) y el Ministerio de Ciencia e Innovación (proyecto CTM200908477). María Gómez fue financiada por una beca del Ministerio de Educación. Cristina Lazcano ha sido financiada mediante un contrato del programa Ángeles Alvariño (Xunta de Galicia).

\section{LITERATURA CITADA}

Aira, M. \& J. Domínguez. 2009. Microbial and nutrient stabilization of two animal manures after the transit through the gut of the earthworm Eisenia fetida. Journal of Hazardous Materials. 161: 12341238.

Aira, M., F. Monroy, J. Domínguez \& S. Mato. 2002. How earthworm density affects microbial biomass and activity in pig manure. European Journal of Soil Biology. 38: 7-10.

Aira, M., F. Monroy \& J. Domínguez. 2006. Eisenia fetida (Oligochaeta, Lumbricidae) activates fungal growth, triggering cellulose decomposition during vermicomposting. Microbial Ecology. 52: 738-746.

Aira, M., F. Monroy \& J. Domínguez. 2007a. Eisenia fetida (Oligochaeta: Lumbricidae) modifies the structure and physiological capabilities of microbial communities improving carbon mineralization during vermicomposting of pig manure. Microbial Ecology. 54: 662-671.

Aira, M., F. Monroy \& J. Domínguez. 2007b. Microbial biomass governs enzyme activity decay during aging of worm-worked substrates through vermicomposting. Journal of Environmental Quality. 36: 448-452.

Atiyeh, R. M., J. Domínguez, S. Subler \& C.A. Edwards. 2000. Biochemical changes in cow manure processed by earthworms (Eisenia andrei) and their effects on plant growth. Pedobiologia. 44: 809-724.

Benítez, E., H. Sainz, R. Melgar \& R. Nogales. 2002. Vermicomposting of a lignocellulosic waste from olive oil industry: a pilot scale study. Waste Management Research. 20: 134-142.

Benítez, E., H. Sainz \& R. Nogales. 2005. Hydrolytic enzyme activities of extracted humic substances during the vermicomposting of a lignocellulosic olive waste. Bioresouce Technology. 96: 785-790.

Bertran, E., X. Sort, M. Soliva \& I. Trillas. 2004. Composting winery waste: sludges and grape stalks. Bioresource Technology. 95: 203-208.

Diaz, M. J., E. Madejón, F. López, R. López \& F. Cabrera. 2002. Optimization of the rate vinasse/ grape marc for co-composting process. Process Biochemistry. 37: 1143-1150.

Dick, W. A., D. Breakwill \& R. Turco. 1996. Soil enzyme activities and biodiversity measurements as integrating biological indicators. Pp. 247-272. In: J. W. Doran and A. I. Jones (Eds.). Handbook of methods for assessment of soil quality. SSSA, Madison.

Domínguez, J. \& C. A. Edwards. 1997. Effects of stocking rate and moisture content on the growth and maturation of Eisenia andrei (Oligochaeta) in pig manure. Soil Biology \& Biochemistry. 29: 743-746.

Domínguez, J. 2004. State of the art and new perspectives in vermicomposting research. Pp. 401-425. In: C. A. Edwards (ed). Earthworm ecology. CRC Press. Boca Raton. 
Gómez-Brandón et al.: Papel de las lombrices de tierra en la degradación del bagazo de uva

Domínguez, J., M. Aira \& M. Gómez-Brandón 2010. Vermicomposting: earthworms enhance the work of microbes. Pp. 93-114. In: H. Insam, I. Franke-Whittle and M. Goberna (Eds.). Microbes at work: from wastes to resources. Springer-Verlag, Berlin Heildelberg.

Doube, B. M. \& G. G. Brown. 1998. Life in a complex community: functional interactions between earthworms, organic matter, microorganisms, and plant growth. Pp. 179-211. In: C. A. Edwards (Ed.). Earthworm ecology. St. Lucie Press, Boca Raton.

Edwards, C. A. 1988. Breakdown of animal, vegetable and industrial organic wastes by earthworms. Pp. 21-31. In: C. A. Edwards and E. F. Neuhauser (Eds.). Earthworms in waste and environmental management. SPB Academic Publishing, The Hague.

Edwards, C. A. \& P. J. Bohlen. 1996. Biology and ecology of earthworms. Chapman and Hall, London.

Fernández-Bayo, J. D., R. Nogales \& E. Romero. 2007. Improved retention of imidacloprid (Confidor $\left.{ }^{\circledR}\right)$ in soils by adding vermicompost from spent grape marc. Science of the Total Environment. 378: 95-100.

Ferrer, J., G. Paez, Z. Marmol, E. Ramones, C. Chandler, M. Marin \& A. Ferrer. 2001. Agronomic use of biotechnologically processed grape wastes. Bioresource Technology. 76: 39-44.

Flavel, T. C., D. V. Murphy, B. M. Lalor \& I. R. P. Fillery. 2005. Gross N mineralization rates after application of composted grape marc of soil. Soil Biology \& Biochemistry. 37: 1397-1400.

Frostegård, Å. \& E. Bååth. 1996. The use of phospholipid fatty acid analysis to estimate bacterial and fungal biomass in soil. Biology \& Fertility of Soils. 22: 59-65.

Goering, H. K. \& P. J. Van Soest. 1970. Forage fiber analysis. Agriculture Handbook 379. Agricultural Research Service, Washington, DC.

Hayano, K. 1986. Cellulase complex in tomato field soil: induction, localization and some properties. Soil Biology \& Biochemistry. 18: 215-219.

Inbar, Y., Y. Chen \& Y. Hadar. 1991. Carbon-13 CPMAS NMR and FTIR spectroscopic analysis of organic matter transformations during composting of solid wastes from wineries. Soil Science. 152: 272-282.

Inbar, Y., Y. Hadar \& Y. Chen. 1992. Characterization of humic substances formed during the composting of solid wastes from wineries. Science of the Total Environment. 113: 35-48.

Ladd, J. N. \& J. H. A. Butler. 1972. Short-term assays of soil proteolytic enzymes activities using proteins and dipeptide derivatives as substrates. Soil Biology \& Biochemistry. 4: 19-30.

Lee, K.E. 1985. Earthworms, their ecology and relationships with soils and land use. Academic Press, Sydney.

Nogales, R., C. Cifuentes \& E. Benítez. 2005. Vermicomposting of winery wastes: a laboratory study. Journal of Environmental Science and Health, Part B: Pesticides, Food Contaminants, and Agricultural Wastes. 40: 659-673.

Romero, E., C. Plaza, N. Senesi, R. Nogales \& A. Polo. 2007. Humic acid- like fractions in raw and vermicomposted winery and distillery wastes. Geoderma. 139: 397-406.

Schinner, F. \& W. Von Mersi. 1990. Xylanase-, CM-cellulase-and invertase activity in soil: an improved method. Soil Biology \& Biochemistry. 22: 511-515.

Vance, E. D., P. C. Brookes \& D. S. Jenkinson. 1987. An extraction method for measuring soil microbial biomass C. Soil Biology \& Biochemistry. 19: 703-707.

Young, J. C. 1995. Microwave-assisted extraction of the fungal metabolite ergosterol and total fatty acids. Journal of Agricultural and Food Chemistry. 43: 2904-2910. 